\title{
STRONG RADIATIVE CORRECTIONS TO STRANGENESS-CHANGING PROCESSES IN THE PRESENCE OF RIGHT-HANDED CURRENTS
}

\author{
I.I. BIGI \\ Institut far Theoretische Physik E, RWTH Aachen, D 5100 Aachen, Fed. Rep. Germany \\ and \\ J.M. FRÈRE \\ Physics Department, University of Michigan, Ann Arbor, MI 48109, USA
}

Received 16 June 1983

\begin{abstract}
In the presence of weak right handed currents the GIM suppression becomes less efficient in the effective $\Delta S=2$ operator. Therefore new features arise and have to be taken into account when applying strong radiative corrections. We determine these QCD corrections to the $\Delta S=2$ operator on the leading log level and (in contrast to the standard model) find a sizeable enhancement. If one ascribes $C P$ violation to the presence of right-handed currents, then the parameter $\epsilon$ will enjoy the same enhancement. We show however that $\epsilon^{\prime}$ is increased in a similar way; the ratio $\epsilon^{\prime} / \epsilon$ is therefore not significantly affected.
\end{abstract}

1. Introduction. Previous studies have shown us that QCD short distance corrections can prove to be very significant in weak processes involving righthanded currents $[1,2]$. Since $\mathrm{K}^{0}-\overline{\mathbf{K}}^{0}$ mixing has recently been advocated as a very sensitive probe for the presence of right handed currents [3] it is important to study the radiative QCD corrections to this process. Our analysis is also motivated by the wellknown possible relevance of right-handed currents for $C P$ violation.

We will see below that strong radiative corrections do affect $\mathrm{K}^{0}-\overline{\mathrm{K}}^{0}$ mixing; however they will not affect significantly the estimate of $\epsilon^{\prime} / \epsilon$ in those models.

The paper will be organized as follows: in section 2 we will discuss $\mathrm{K}^{0}-\mathbf{K}^{0}$ mixing in the presence of right handed currents and gluon exchanges spelling out some of the technical details involved when applying renormalization arguments; in section 3 we summarize briefly $C P$ violation mechanisms in those weak models and show how in particular the ratio $\epsilon^{\prime} / \epsilon$ is affected by strong radiative corrections; section 4 contains our conclusions.
2. The contribution of right handed currents to $\mathrm{K}^{0}-\overline{\mathrm{K}}^{0}$ mixing has been shown to be greatly enhanced for three reasons [3]:

(i) There are more graphs.

(ii) Matrix element enhancements due to PCAC generate factors $\sim\left(m_{\mathrm{K}} / m_{\mathrm{S}}\right)^{2}$ where $m_{\mathrm{K}}\left(m_{\mathrm{S}}\right)$ denotes the kaon (strange quark) mass.

(iii) a less efficient GIM suppression leads to large logarithmic contributions $\sim \log \left(M_{\mathrm{W}}^{2} / m_{\mathrm{c}}^{2}\right)$.

This last reason leads to the importance of momenta $m_{\mathrm{c}}^{2}<p^{2}<M_{\mathrm{w}}^{2}$ when integrating over the intemal loop in the familiar box diagram. This is in marked contrast to the standard model where momenta $p^{2} \sim m_{\mathrm{c}}^{2}$ or $p^{2} \sim m_{\mathrm{t}}^{2}$ are dominant. This circumstance, while making renormalization group arguments more reliable, forces us at the same time into a more careful identification and subsequent integration of QCD logarithms. A similar situation was encountered and nicely discussed by Vysotskii in the context of the standard model [4] when a mixed top and charm exchange is considered inside the box dia. gram.

We will limit our discussion to the four-quark 
model since it already exposes all the new features and inclusion of the top quark does not significantly modify the numerical results.

To calculate strong radiative corrections to the ef fective $\Delta S=2$ operator one has first to identify those graphs which lead to large logarithms when applying QCD corrections. Using as usual the Landau gauge for gluons to reduce the number of relevant diagrams we find three types of such graphs as shown in figs. 1a$1 \mathrm{c}$, which will now be discussed in turn. (One easily checks that no contribution arises from the exchange of a gluon between two s or two d lines.)

The corrections due to diagrams as shown in fig. la can be interpreted as integrals involving multiplicatively renormalized local operators $O_{\mathrm{L}}^{ \pm}$and $O_{\mathrm{R}}^{ \pm}$which are familiar from the standard model [1]:

$$
\begin{aligned}
& \left.\left.O_{\left[\begin{array}{l}
\mathrm{L} \\
\mathrm{R}
\end{array}\right]}^{ \pm}(x)=\overline{\mathrm{u}}_{\left[{ }_{\mathrm{R}}^{\mathrm{L}}\right]} \gamma_{\mu}{ }_{\left[{ }_{\mathrm{R}}^{\mathrm{L}}\right.}^{\mathrm{L}}\right]^{\overline{\mathrm{d}}}{ }_{[\mathrm{R}}^{\mathrm{L}}\right]^{\gamma_{\mu}}{ }^{\left.\overline{\mathrm{u}}_{[\mathrm{R}}^{\mathrm{L}}\right]}
\end{aligned}
$$

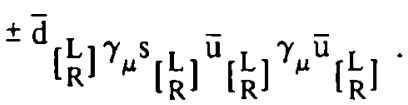

Large logarithms are produced by integrating the gluon momentum $q^{2}$ from the quark momentum $p^{2}$ up to $M_{\mathrm{L}}^{2}$ or $M_{\mathrm{R}}^{2}$ respectively.

The graph in fig. $1 \mathrm{~b}$ contains a renormalization of the charm quark mass which - after an appropriate subtraction - can give a potentially large logarithm for $q^{2}>p^{2}$.

Finally, the gluon loop in fig. Ic leads to the same anomalous dimension as a mass renormalization and can give a large logarithmic contribution for $\mu^{2}<q^{2}$ $<p^{2}$, where $\mu$ is the (virtual) mass of the external

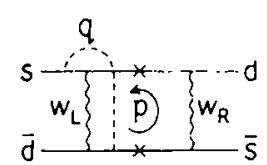

a

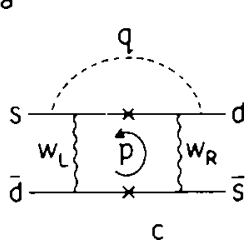

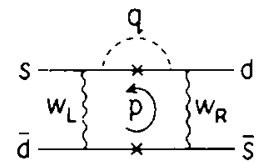

b
Fig. 1. The non-vanishing one-toop gluon corrections to the $\Delta S=2$ operator containing $W_{\mathrm{L}}$ and $\mathrm{W}_{\mathrm{R}}$ exchange. quark fields. Due to the weaker GIM suppression the whole range $M_{\mathrm{L}}^{2}>p^{2}>m_{\mathrm{c}}^{2}$ with $p$ being the internal quark momentum contributes significantly to the in. tegral; we will the refore neglect the range $m_{\mathrm{c}}^{2}>p^{2}>$ $\mu^{2}$ in what follows since it will be of minor numerical importance. We use the following notation: (We pull out a factor of $\ln \left(M_{L}^{2} / m_{c}^{2}\right)$ to factorize the non-radiative correction result.)

$$
\begin{aligned}
& \mathcal{L}_{\mathrm{eff}}^{\mathrm{LR}}(\Delta S=2)=\left(G_{\mathrm{F}}^{2} / 4 \pi^{2}\right) 8 m_{\mathrm{c}}^{2} \\
& \quad \times \sin \theta_{\mathrm{L}} \sin \theta_{\mathrm{L}} \cos \theta_{\mathrm{L}} \cos \theta_{\mathrm{R}} \\
& \quad \times\left(M_{\mathrm{L}}^{2} / M_{\mathrm{R}}^{2}\right) \ln \left(M_{\mathrm{L}}^{2} / M_{\mathrm{R}}^{2}\right) I_{\mathrm{LR}}\left\{O_{\mathrm{LR}}+O_{\mathrm{RL}}\right\},
\end{aligned}
$$

where $\theta_{\mathrm{L}}, \theta_{\mathrm{R}}$ are the left and right-handed Cabibbo angles.

$$
\begin{aligned}
& \left.O_{\mathrm{LR}[\mathrm{RL}]}=\left(\overline{\mathrm{d}}_{\mathrm{R}[\mathrm{L}]}\right]_{\mathrm{L}[\mathrm{R}]}\right)\left(\overline{\mathrm{d}}_{\mathrm{L}[\mathrm{R}]} \mathrm{s}_{\mathrm{R}[\mathrm{L}]}\right), \\
& I_{\mathrm{LR}}=\frac{3}{2} I_{\mathrm{LR}}^{++}-\frac{1}{2} I_{\mathrm{LR}}^{+-}-\frac{1}{2} I_{\mathrm{LR}}^{-+}+\frac{1}{2} I_{\mathrm{LR}}^{-} .
\end{aligned}
$$

The $I_{\mathrm{LR}}^{ \pm \pm}$contain the QCD corrections and the bare contribution. Using the well known expression for the QCD coupling

$$
\alpha_{\mathrm{s}}\left(R^{2}\right)=\left[12 \pi /\left(33-2 N_{\mathrm{f}}\right)\right] / \log \left(k^{2} / \Lambda^{2}\right)\left(k^{2} \gg \Lambda^{2}\right),
$$

and applying the renormalization group in the usual fashion to exponentiate one-loop results we obtain

$$
\begin{aligned}
& I_{\mathrm{LR}}^{i j_{\mathrm{R}}}=\left[\ln \left(M_{\mathrm{L}}^{2} / m_{\mathrm{c}}^{2}\right)\right]^{-1} \int_{m_{\mathrm{c}}^{2}}^{M_{\mathrm{L}}^{2}} \frac{p^{2}}{\left(p^{2}-m_{\mathrm{c}}^{2}\right)^{2}} \\
& \quad \times\left[\alpha_{\mathrm{s}}\left(p^{2}\right) / \alpha_{\mathrm{s}}\left(M_{\mathrm{L}}^{2}\right)\right]^{\gamma_{i}\left[\alpha_{\mathrm{s}}\left(p^{2}\right) / \alpha_{\mathrm{s}}\left(M_{\mathrm{R}}^{2}\right)\right]^{\gamma_{j}}} \\
& \quad \times\left[\alpha_{\mathrm{s}}\left(p^{2}\right) / \alpha_{\mathrm{s}}\left(\mu^{2}\right)\right]^{2 \gamma_{\mathrm{m}}}\left[\alpha_{\mathrm{s}}\left(p^{2}\right) / \alpha_{\mathrm{s}}\left(m_{\mathrm{c}}^{2}\right)\right]^{2 \gamma_{\mathrm{m}} \mathrm{d} p^{2}},
\end{aligned}
$$

with

$$
\begin{aligned}
& i, j= \pm, \quad \gamma_{+}=-2 / b, \quad \gamma_{-}=4 / b, \\
& 2 \gamma_{\mathrm{m}}=-8 / b, \quad b=11-\frac{2}{3} N_{\mathrm{f}} .
\end{aligned}
$$

Since the integrals over $p$ are logarithmically divergent for $M_{\mathrm{L}} \rightarrow \infty$ already in the absence of gluon corrections, one is not allowed to pull the factors $\alpha_{s}\left(p^{2}\right)$ $\propto\left(\log p^{2}\right)^{-1}$ outside the integral by writing them as $\alpha_{\mathrm{s}}\left(m_{\mathrm{c}}^{2}\right)$. Performing the integrations in (2.6) one finds: 


$$
\begin{aligned}
I_{\mathrm{LR}} & =\left[\alpha_{\mathrm{s}}\left(\mu^{2}\right) \alpha_{\mathrm{s}}\left(m_{\mathrm{c}}^{2}\right) / \alpha_{\mathrm{s}}^{2}\left(M_{\mathrm{L}}^{2}\right)\right]^{24 / 25} \\
& \times\left\{\frac{15}{34}\left[\alpha_{\mathrm{s}}\left(M_{\mathrm{R}}^{2}\right) / \alpha_{\mathrm{s}}\left(M_{\mathrm{L}}^{2}\right)\right]^{6 / 25}\left(1-\left[\alpha_{\mathrm{s}}\left(M_{\mathrm{L}}^{2}\right) / \alpha_{\mathrm{s}}\left(m_{\mathrm{c}}^{2}\right)\right]^{17 / 5}\right)\right. \\
& -\frac{25}{134}\left(1-\left[\alpha_{\mathrm{s}}\left(M_{\mathrm{L}}^{2}\right) / \alpha_{\mathrm{s}}\left(m_{\mathrm{c}}^{2}\right)\right]^{67 / 25}\right) \\
& \times\left(\left[\alpha_{\mathrm{s}}\left(M_{\mathrm{R}}^{2}\right) / \alpha_{\mathrm{s}}\left(M_{\mathrm{L}}^{2}\right)\right]^{-12 / 25}+\left[\alpha_{\mathrm{s}}\left(M_{\mathrm{R}}^{2}\right) / \alpha_{\mathrm{s}}\left(M_{\mathrm{L}}^{2}\right)\right]^{6 / 25}\right) \\
& +\frac{25}{95}\left[\alpha_{\mathrm{s}}\left(M_{\mathrm{R}}^{2}\right) / \alpha_{\mathrm{s}}\left(M_{\mathrm{L}}^{2}\right)\right]^{-12 / 25} \\
& \left.\times\left(1-\left[\alpha_{\mathrm{s}}\left(M_{\mathrm{L}}^{2}\right) / \alpha_{\mathrm{s}}\left(m_{\mathrm{c}}^{2}\right)\right]^{49 / 25}\right)\right\} \\
& \times \ln \left(M_{\mathrm{L}}^{2} / \mu^{2}\right) / \ln \left(M_{\mathrm{L}}^{2} / m_{\mathrm{c}}^{2}\right)
\end{aligned}
$$

where we have assumed four active flavors.

Allowing the QCD parameter $\Lambda$ to vary between $100 \mathrm{MeV}$ - as suggested by QCD sum rules and by the $\Delta I=1 / 2$ rule - and $250 \mathrm{MeV}$ - as extracted from deep inelastic data - we obtain

$I_{\mathrm{LR}}=(3 \ldots 8)$,

where the smaller value of $\Lambda$ corresponds to the smaller numerical coefficient. These values are quoted for $M_{\mathrm{R}} \sim 10 M_{\mathrm{L}}$ but do not depend strongly on this ratio. Thus we find that gluon corrections produce a sizeable if not large enhancement of the effective $\Delta S=2$ operator.

So far we have neglected contributions due to $W_{L}-W_{R}$ mixing (fig. 2); the graph in fig. 2a is subject only to the weaker GIM suppression as it was discussed for the diagrams in fig. 1 and is therefore more im. portant than the graph in fig. $2 \mathrm{~b}$ experiencing the full GIM suppression. We feel justified in ignoring these two contributions to the $\mathrm{K}^{0}-\overline{\mathrm{K}}^{0}$ transition since they are of second order in the $\mathrm{W}_{\mathrm{L}}-\mathrm{W}_{\mathrm{R}}$ mixing angle $\xi$ on which an upper bound of at most a few percent can be placed [2].

3. Although $W_{L}-W_{R}$ mixing cannot yield significant contributions to the $\mathrm{K}^{0}-\overline{\mathrm{K}}^{0}$ transition, it could prove very important for the phenomenon of $C P$ vio-
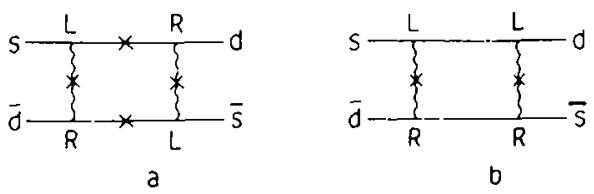

Fig. 2. Contributions to the $\Delta S=2$ operator due to $W_{L}-W_{R}$ mixing. lation, in particular in the ratio $\epsilon^{\prime} / \epsilon$ measuring on shell versus off-shell $C P$ violation in the $\mathrm{K}^{0}-\overline{\mathrm{K}}^{0}$ system. It has indeed been argued that both algebraic $[5,6]$ and "kinematical" $[6,7]$ effects can be employed to turn left-right symmetric models effectively in to superweak models.

The "kinematical" argument is essentially based on the above mentioned enhancement of $\Delta S=2$ contributions in left-right models, already before gluon corrections are included: any imaginary part due to phases appearing in the right-handed sector is enhanc. ed exactly like the real part of the graph. $\Delta S=1$ transitions in the absence of $W_{L}-W_{R}$ mixing enjoy no such enhancements; assuming only one phase to be present (or that all phases are of the same order) one can then estimate for the value of $\epsilon^{\prime} / \epsilon$ :

$$
\begin{aligned}
\epsilon^{\prime} / \epsilon & =2^{-1 / 2}\left|A_{0}\right|^{-1} \operatorname{Im} A_{2} \\
& =2^{-1 / 2}\left(\left|A_{2}\right| /\left|A_{0}\right|\right)\left|A_{2}\right|^{-1} \operatorname{Im} A_{2}=2^{-1 / 2} \frac{1}{20}(\mathrm{EF})^{-1},
\end{aligned}
$$

where $A_{I}$ denote the amplitudes leading to isospin $I$ in the final state and EF stands for the enhancement factor present in the $\Delta S=2$ matrix element. Using the calculated value [3] EF $\sim 400$ one gets

$\epsilon^{\prime} / \epsilon \sim 10^{-4}$.

It has been advocated the $W_{L}-W_{R}$ mixing, which besides its angle $\xi$ could also contain a complex phase, would not change this almost superweak prediction very much $[6,7]$. It is this claim we want to analyze in more detail now. In particular, ref. [7] estimates $\epsilon^{\prime} / \epsilon \leqslant 10^{-3}$ in a specific model, before QCD corrections.

The relevant operator is shown in fig. 3 . Its real part vanishes if the Cabibbo angles in the left - and in the right-handed sector are the same; however if there is a complex phase $\phi$ present, the imaginary parts add up. Inspection of the diagram reveals that decays leading to $a \pi^{0}$ in the final state will enjoy a matrix

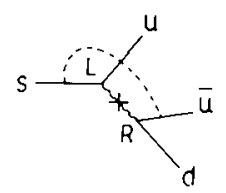

Fig. 3. $\Delta S=1$ operator due to $\mathrm{W}_{\mathrm{L}}-\mathrm{W}_{\mathrm{R}}$ mixing with gluon correction. 
element enhancement $F_{1}$ due to PCAC with

$F_{1}=m_{\pi}^{2} / \frac{1}{2}\left(m_{\mu}+m_{\mathrm{d}}\right) m_{\mathrm{s}} \sim 20$.

$\operatorname{Im} A_{2}$ (the $\Delta I=3 / 2$ decay amplitude) and thus $\epsilon^{\prime}$ will be increased proportionally. This was already recognized in ref. [7] and leads to the estimate 3.2. QCD corrections to this operator, as indicated in fig. 3 , are very important. As pointed out by Altarelli and Maiani [1] the multiplicatively renormalized operators are

$$
\begin{gathered}
O_{1}=2\left[\sin \theta_{L} \cos \theta_{R}\left(\overline{\mathrm{d}}_{\mathrm{R}} \mathrm{s}_{\mathrm{L}}\right)\left(\overline{\mathrm{u}}_{\mathrm{L}} \mathrm{u}_{\mathrm{R}}\right)\right. \\
\left.+\sin \theta_{\mathrm{R}} \cos \theta_{\mathrm{L}}\left(\overline{\mathrm{d}}_{\mathrm{L}} \mathrm{s}_{\mathrm{R}}\right)\left(\overline{\mathrm{u}}_{\mathrm{R}} \mathrm{u}_{\mathrm{L}}\right)\right],
\end{gathered}
$$

and

$$
\begin{aligned}
O_{8} & =12\left[\sin \theta_{\mathrm{L}} \cos \theta_{\mathrm{R}}\left(\overline{\mathrm{d}}_{\mathrm{R}} \frac{1}{2} \lambda_{i} \mathrm{~s}_{\mathrm{L}}\right)\left(\overline{\mathrm{u}}_{\mathrm{L}} \frac{1}{2} i \mathrm{u}_{\mathrm{R}}\right)\right. \\
& \left.+\sin \theta_{\mathrm{R}} \cos \theta_{\mathrm{L}}\left(\overline{\mathrm{d}}_{\mathrm{L}} \frac{1}{2} \lambda_{i} \mathrm{~s}_{\mathrm{R}}\right)\left(\overline{\mathrm{u}}_{\mathrm{R}} \frac{1}{2} \lambda_{i} \mathrm{u}_{\mathrm{L}}\right)\right],
\end{aligned}
$$

with anomalous dimensions $\gamma_{1}=24 / 25$ and $\gamma_{8}=-3 /$ 25 ; the effective lagrangian then become

$$
\begin{aligned}
& \mathcal{L}_{\mathrm{eff}}^{\mathrm{LR}}=2^{-1 / 2} G_{\mathrm{F}}(-\sin \xi) \\
& \quad \times\left\{\frac{1}{3}\left[\alpha_{\mathrm{s}}\left(\mu^{2}\right) / \alpha_{\mathrm{s}}\left(M_{\mathrm{L}}^{2}\right)\right]^{\gamma_{1}} O_{1}\right. \\
& \left.\quad+\frac{1}{3}\left[\alpha_{\mathrm{s}}\left(\mu^{2}\right) / \alpha_{\mathrm{s}}\left(M_{\mathrm{L}}^{2}\right)\right]^{\gamma_{8}} O_{8}\right\} .
\end{aligned}
$$

Thus there is a second enhancement factor $F_{2} \simeq 3 \ldots 8$ due to QCD corrections. The combined effect of $F_{1}$ and $F_{2}$ would bring the estimate of $\epsilon^{\prime} / \epsilon$, eq. (3.2), in to the percent range - were it not for the QCD corrections which also enhance $\epsilon$ as discussed in section 2. Therefore we finally estimate

$$
\left|\epsilon^{\prime} / \epsilon\right|_{\text {LR+QCD }} \lesssim \text { few } \times 10^{-3} \text {. }
$$

4. We can draw three main conclusions from our results presented in the two preceding sections:

(1) QCD radiative corrections are very sizeable when righthanded weak currents are included.

(2) They increase the strength of the $\Delta S=2$ effec. tive operator thus making the bound on $M_{\mathrm{W}_{\mathrm{R}}}$ obtained from it [3] even more restrictive.

(3) Although they increase estimates both of $\epsilon^{\prime}$ and of $\epsilon$ considerably they do not modify their ratio very much. Therefore mech anisms evoked in the weak sector to ensure

$\left|\epsilon^{\prime} / \epsilon\right|_{\text {LR }} \lesssim$ few $\times 10^{-3}$,

will still work even after inclusion of QCD corrections.

We have benefitted from discussions with A. Sanda, A. Sirlin and A. Soni. We are very grateful to T. Goldman and $\mathrm{H}$. Haber who organized such a fine TSIMES meeting in Santa Cruz where we started this study. We also especially thank Tony Sanda and the Rockefeller University for their kind hospitality.

\section{References}

[1] G. Altarelli and L. Maiani, Phys. Lett. 52B (1974) 351.

[2] I.I. Bigi and J.-M. Frère, Phys. Lett. 110B (1982) 255.

[3] G. Beall, M. Bander and A. Soni, Phys. Rev. Lett. 48 (1982) 848.

[4] M.I. Vysotskii, Sov. J. Nucl. Phys. 31 (1980) 797; see also F. Gilman and M.B. Wise, Phys. Lett. 93B (1980) 129.

[5] R. Mohapatra and J. Pati, Phys. Rev. D 11 (1975) 566.

[6] G. Branco, J.-M. Frère and J.-M. Gerard, CERN TH 3406, to be published in Nucl. Phys. B.

[7] D. Chang, Carnegie-Mellon preprint, CMU-HEG 82-8. 